\section{Chiari type I malformation with cervicothoracic syringomyelia subterfuge as flail arm syndrome}

\author{
Zhi Gang Lan, 1 Seidu A. Richard, 1-3 \\ Jiagang Liu, ${ }^{1}$ Chao You ${ }^{1}$ \\ 1Department of Neurosurgery, West \\ China Hospital, Sichuan University, \\ Sichuan; 2Department of Immunology, \\ Jiangsu University, Jiangsu, China; \\ 3Department of Surgery, Volta Regional \\ Hospital, Ho, Ghana-West Africa
}

\begin{abstract}
Chiari type I malformation with cervicothoracic syringomyelia although very common in clinical practice usually in children can progress slowly and mimic muscular dystrophies in adulthood. We present a rare adult case of Chiari type I malformation with cervicothoracic syringomyelia subterfuge as Flail arm syndrome. A 44year-old man was diagnosed with congenital type I Chiari malformation with cervicothoracic syringomyelia about 21 years ago without surgery. His health status deteriorated over the years until 21 days prior to presentation when he had severe pain in the right knee. In his upper limbs, he had bilateral corresponding severe weakness of $0 / 5$ proximal strength and $0 / 5$ strength in his distal muscles. Magnetic resonance imaging (MRI) revealed an enlargement of the spinal cord from C1-C4 level with a mass that appeared hypo-dense on $\mathrm{T} 1$ and hyperdense on T2. Syringomyelia is a potentially serious neurologic condition that can mimic other neuromuscular disorders. Early detection and diagnosis with MRI is crucial to avoid irreversible neurological complications. We suggest that whether asymptomatic or symptomatic, decompressive surgery should be carried out to allow for free flow of cerebrospinal fluid thereby improving the quality of life for the patient.
\end{abstract}

\section{Introduction}

Syringomyelia occurs when a cyst, typically made up of excess cerebrospinal fluid (CSF), cumulates inside the spinal cord either within the parenchyma as a focal dilation of the central canal. ${ }^{1-5}$ Chiari malformation type I is a form of non-communicating syringomyelia. ${ }^{3-6}$ Flail arm syndrome (FAS), also referred to as brachial amyotrophic diplegia or man-in-barrel syn- drome, is a gradually progressive sporadic motor neuron disorder, depicted by severe flaccid paralysis and muscle wasting in both arms symmetrically, but comparatively sparing the legs and bulbar parts, and scarcely signs of upper motor neuron lesions.7-9 The onset of syringomyelia although commonly chronic and gradually progressive, very few case advanced into adulthood unnoticed. The severe progressive adult type is most likely to mimic muscular dystrophy's ${ }^{4}$. Cerebellar tonsilar ectopia bulging through the foramen magnum is frequently the imaging findings in patients with this condition. $3,4,10$ The gold standard of treatment for this disorder is surgery and the choice of surgical approaches for Chiari type I, depending on the surgeon's understanding of the pathophysiology of the disease manifestation. ${ }^{1,4}$ In most cases the pathology is situated at the level of the foramen magnum leading to disparities in the contents and the volume of the posterior fossa. We present an adult case of congenital type I Chiari malformation with cervicothoracic syringomyelia subterfuge as Flail arm syndrome and review of literature.

\section{Case Report}

A 44-year-old man presented with worsening chronic motor symptoms for 21 years. He is a known case of congenital type I Chiari malformation with cervicothoracic syringomyelia diagnosed about 21 years ago without surgery. He was not able to access surgery from the time of the initial diagnosis (21 years ago) until he presents at our facility because of financial constraints. He health status deteriorated over the years until 21 days prior to presentation when he had severe pain in the right knee. The right knee pain is aggravated by walking, climbing stairs or in sitting and relieved by lying in bed. There was no associated swelling, no neck pains, backaches or abdominal pains. He denied having dysphagia, cough, difficulty in breathing or palpitations. All other systems were normal. Physical examination demonstrated no face weakness, numbness, sensory deficits, unusual reflexes, muscle fasciculations, as well as upper motor neuron signs. Examination of limbs revealed severe atrophy of his bilateral forearm flexor and extensor muscle groups with his lower extremities spared (Figure 1A). In the upper limbs, he had bilateral corresponding severe weakness of $0 / 5$ proximal strength and $0 / 5$ strength in his distal muscles (Figure 1B). He could only lift his hands with support. He had $5 / 5$ strength in his bilateral hip extensors and flexors and $5 / 5$ strength in bilateral knee extensors and
Correspondence: Jiagang Liu, Department of Neurosurgery, Post-Graduate Training Centre, West China Hospital, Sichuan University, 37 Guo Xue Xiang Road, Chengdu, Sichuan, 610041, China.

Tel: +86-028-85422488 - Fax: +86-28-85422490.

E-mail: vipneurology@yeah.net

Key words: Chiari type I malformation; Cervicothoracic; Syringomyelia; Subterfuge; Flail arm syndrome.

Ethics approval and consent to participate: The ethical committee of the hospital fully approved our case study. The patient was informed about our intention to involve him in a case study and he agreed to partake in the study. He signed the concern form before the operation was carried out according to all surgical protocols.

Consent for publication: the patient was dually informed about our intention to publish his case and she fully concerted to the use of these documents. The hospital also concerted to the use of this information for publication.

Conflict of interest: the authors declare no potential conflict of interest.

Contributions: all the authors contributed equally to the manuscript design and writing.

Received for publication: 30 July 2017.

Revision received: 7 August 2017.

Accepted for publication: 21 August 2017.

This work is licensed under a Creative Commons Attribution NonCommercial 4.0 License (CC BY-NC 4.0).

(C) Copyright Z.G. Lan et al., 2017

Licensee PAGEPress, Italy

Neurology International 2017; 9:7336 doi:10.4081/ni.2017.7336

could walk about except for the right knee pain, which limited his movement in the ward. All routine laboratory investigations were essentially normal. Electrophysiological studies reveal bilateral absence of median, ulnar and radial nerves compound muscle action potentials (CMAP) with slightly abnormal sensory nerve action potential (SNAP) at the upper limbs. However, the all the nerves on the lower limbs have normal CMAPs and normal SNAPs. Electromyography (EMG) of his upper extremities revealed of diffuse chronic neurogenic changes in almost all the nerves but normal in all the nerves in the lower extremities.

Preoperative MRI done revealed an enlargement of the spinal cord from $\mathrm{C} 1-\mathrm{C} 4$ level. Within the spinal cord is a mass that appears hypo-intense on $\mathrm{T} 1$ and hyper- 
intense on T2. These findings are consistent with Chiari malformation type I with prominent syringomyelia (Figure $2 \mathrm{~A}$ and B). MRI of lumbar vertebral and adjacent structures showed no abnormalities. There is no disc space narrowing, no abnormal vertebral body and no cord stenosis. The patient was the scheduled for suboccipital decompression of the syringomyelia.

The patient was on park-bench position after general anesthesia with the surgical incision area marked. A straight median incision was made from the occipital protuberance up to C3. Subcutaneous tissues and muscle was dissected up the C3. Suboccipital craniectomy and laminectomy of $\mathrm{C} 1$ and $\mathrm{C} 2$ was done using the high-drill and further exposer with a Kerrison rongeur. Resection of the atlanto-occipital dura was done to free the compression. The dura was peeled off with a Y shaped incision and the arachnoid membrane was intact. After securing total hemostasis, the fascia, muscle, subcutaneous tissues and skin was closed in layers. Estimated blood lost was $50 \mathrm{~mL}$. We noticed during surgery that there were no obvious abnormalities on the skin and subcutaneous tissues but however, there was thickening of paraspinal muscle with retracted occipital plane and thickening of atlanto-occipital fascia. The patient was nursed in the recovery word and later to neurosurgery word. Post-operative management was uneventful. His amyotrophy did not improve much while on admission. He was discharged home two weeks after the operation. Outpatient visits were arranged at 3 months' interval. MRI done at the 2-year follow up showing the syringomyelia was shrinked significantly (Figure 2C and D). His amyotrophy remained stable over the years afterwards.

\section{Discussion}

The precise pathogenic link between syringomyelia and Chiari type 1 malformation is still a matter of debate among neurosurgeons and quite a number of authors have proposed various theories as the formation of syrinx. ${ }^{1,3,4}$ Most of the authors have come to the conclusion that malformation of the mesodermal occipital somite is accountable for the tiny nature of the posterior fossa leading to the anomalous CSF flow at the level of the foramen magnum. ${ }^{1,11,12}$ Therefore, most disorders originate from the cervical spinal cord and can enlarge superiorly into the brainstem leading to the formation of syringobulbia or inferiorly into the thoracic and sometimes the lumbar regions. 3,4 Apart from Chairi type 1 malformations, Syringomyelia can

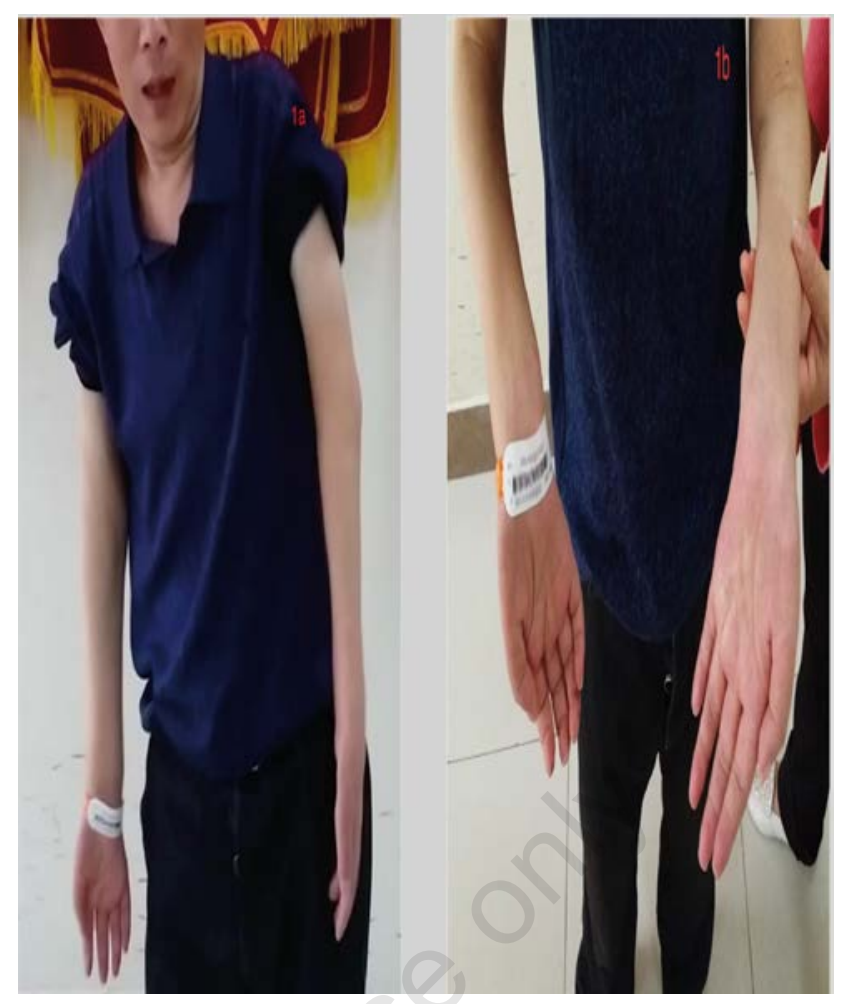

Figure 1. Physical examination showing patient's upper extremities with severe atrophy of forearm extensors (A) and flexors (B).
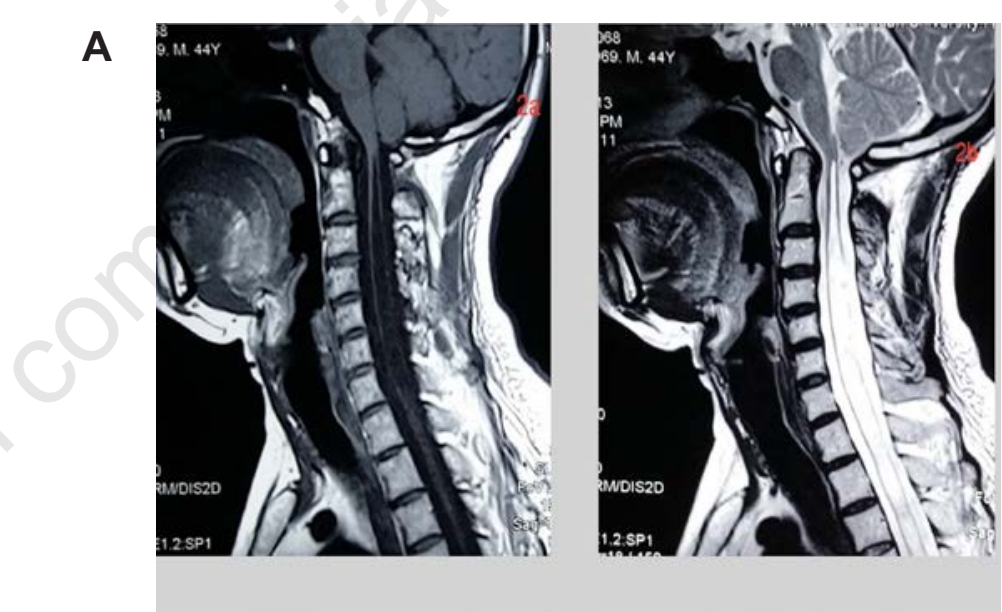

B

C
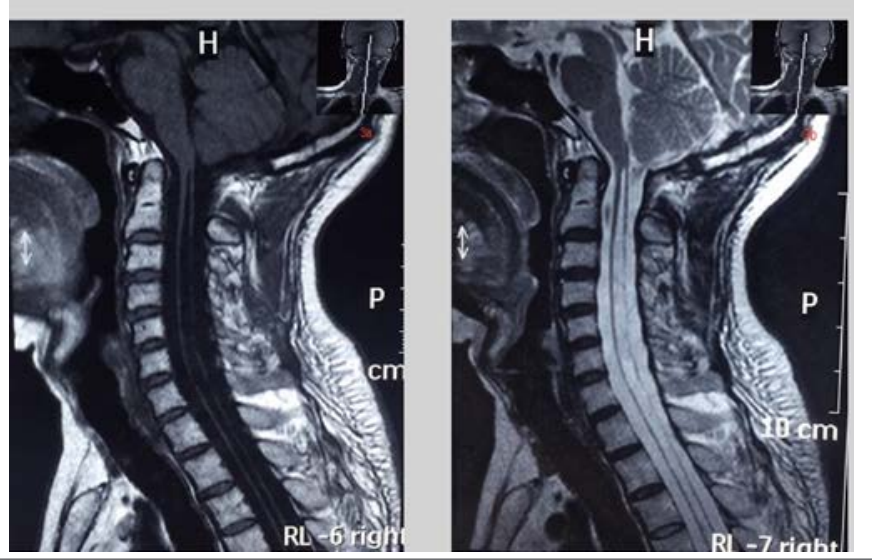

D 
occur together with diverse number of congenital anatomic anomalies as well as acquired structural abnormalities such as scoliosis, spina bifida, tumors and hemorrhage, post-infectious, post-inflammatory and post-traumatic conditions. . $^{5}$

FAS characteristically presents with progressive upper limb weakness and wasting that is often symmetric and proximal, without significant functional involvement of lower limbs or

bulbar muscles. ${ }^{4,8,9} \mathrm{Hu}$ et al used FAS to describe this disorder 9,13 although many other authors have also described the same disorder and given it varies names such as Vulpian-Bernhardt syndrome, $8,9,14$ hangingarm syndrome, $, 9,15$ neurogenic man-in-abarrel syndrome, 8,9 and brachial amyotrophic diplegia. 8,9 The presentation of our case typically mimics the above condition with diverse names. Amyotrophic lateral sclerosis (ALS) is also a degenerative motor neuron disorder, with progressive loss of both upper and lower motor neurons in motor cortex, spinal anterior horn cells and motor neurons in brain stem.7,16 Our patient disease presentation does not correspond to ALS because his lower limbs was spared.

Syringomyelia is general classified based on the pathological and magnetic resonance physiognomies of numerous spinal cord cysts. They are put into five categories: non-communicating, communicating, primary parenchymal cavitations, atrophic cavitations and neoplastic cavitations. ${ }^{4,6}$ The symptomatology of syrinx can be relatively complicated and diverse among patients because of the different syrinx types, the spinal levels involved, the degree of extension as well as the diameter of lesion. ${ }^{4,17}$ The potential permutations of the clinical symptoms comprise of headache, severe segmental central and dysesthetic pain, loss of temperature and pain sensation, down-beating nystagmus, vocal cord dysmotility, urinary frequency and incontinence, stiffness, weakness, and scoliosis.4,17-19 Mora et al noticed central cord syndrome as one of the cardinal presentation of this condition. ${ }^{4}$

MRI is the gold standard for the diagnosis of a Chiari type 1 malformation with or without Syringomyelia. ${ }^{20}$ Patients with Syringomyelia and Chiari type 1 malformation should have MRI of their brain and spine during their evaluation. CAT scan (CT) although valuable does not provide accurate diagnosis of this disorder. It is however the best in visualizing the bone anatomy of the foramen magnum. ${ }^{20} \mathrm{MRI}$ of the brain and spine will tell whether or not a patient has Chiari type 1 malformation and/or syringomyelia, or any other abnormality of the brain that may cause similar descendance of the cerebellar tonsils approximately $3-5 \mathrm{~mm}$ or more below the opening in the bottom of the skull. ${ }^{20-22} \mathrm{~T} 1 \mathrm{~W}$ image produces a more accurate reflection of cyst size than the T2W image, because it is less sensitive to flow effects. Septa in the cyst can best be seen in the T1W image. ${ }^{10}$ Zamel et al. proposed the use of brain stem auditory evoked potentials during posterior fossa decompression to assess functional integrity of the central auditory system during surgery. 23

Surgery is the main treatment for patients with syringomyelia. Nevertheless, not all patients need surgical intervention. Patients who are incidentally diagnosed with MRI studies but do have any clinical manifestation of the disorder are mostly followed with serial imaging in some institutions. Some author thinks these kinds of patients should be operated.1,4 Some authors have indicated that most patients with mild symptoms remain stable and in some instances their disorders unexpectedly disappear on radiology while other are still of the view that such patient should have surgical correction of the anomaly right way to avoid further complications. 4,24 We are of the view that syringomyelia whether asymptomatic or mild should be surgical corrected putting the history of advancement of our case into perspective. Surgery is the usually the required treatment option for patients with obvious symptoms. Decompression to reinstate regular CSF flow is the goal of surgery. Posterior foramen magnum decompression with or without dura opening or anterior foramen magnum decompression and shunting are some of the surgical techniques use by varies authors. $1,4,17$

Navarro et al. are of the view that the best surgical approach to this disorder is to recreate the mesodermal section at the level of the foramen magnum. They indicated that bone and dura should be reconstructed without disrupting the structures emanating from the neuroectoderm, that is the leptomeninges and cerebellum. ${ }^{1}$ This approach would reduce most surgical complications that are associated with CSF release and adhesive arachnoiditis by enlarging the posterior fossa and redirecting the flow of CSF hence minimizing morbidity. ${ }^{1}$ Most authors including Navarro et al agree with the use of suboccipital craniectomy and laminectomy to the level of the tonsillar herniation. They are of the view that after resecting the dura thickened band, careful dissection of the dura is commenced by protecting the inner layer of the dura and avoiding arachnoid laceration. This maneuver releases the pressure on the dura and grant passage of the posterior fossa contents because the dura and bone together provides opposition to the cerebellum.1,25-27 Most patients experience mild improvement to restoration of function after surgical decompression the Chiari malformation depending on the magnitude of their symptoms before surgery. ${ }^{4}$ In adults, the average recurrence rate after decompressive surgery is about $6.7 \%$ in most patience. 4,17

\section{Conclusions}

Syringomyelia is a potentially serious neurologic condition that can mimic other neuromuscular disorders. Early detection and diagnosis with MRI is crucial to avoid irreversible neurological complication. We suggest that whether asymptomatic or symptomatic, decompressive surgery should be carried out to allow for free flow of CSF thereby improving the quality of life for the patient.

\section{References}

1. Navarro R, Olavarria G, Seshadri R, et al. Surgical results of posterior fossa decompression for patients with Chiari I malformation. Child's Nervous Syst 2004;20:349-56.

2. Zhang Z, Chen Y, Chen Y, et al. Chiari I malformation associated with syringomyelia: a retrospective study of 316 surgically treated patients. Spinal Cord 2008;46:358-63.

3. Koyanagi I, Houkin K. Pathogenesis of syringomyelia associated with Chiari type 1 malformation: review of evidences and proposal of a new hypothesis. Neurosurg Rev 2010;33:271-85.

4. Mora JR, Rison RA, Beydoun SR. Chiari malformation type I with cervicothoracic syringomyelia masquerading as bibrachial amyotrophy: a case report. J Med Case Rep 2015;9:11.

5. Stoodley MA, Jones NR, Yang L, et al. Mechanisms underlying the formation and enlargement of noncommunicating syringomyelia: experimental studies. Neurosurg Focus 2000;8:1-7.

6. Brickell KL, Anderson NE, Charleston $\mathrm{AJ}$, et al. Ethnic differences in syringomyelia in New Zealand. J Neurol Neurosurg Psych 2006;77:98991.

7. Yang H, Liu M, Li X, et al. Neurophysiological differences between flail arm syndrome and amyotrophic lateral sclerosis. PloS one 2015;10:e0127601.

8. Katz J, Wolfe G, Andersson P, et al. 
Brachial amyotrophic diplegia A slowly progressive motor neuron disorder. Neurology 1999;53:1071-71.

9. Wijesekera L, Mathers S, Talman P, et al. Natural history and clinical features of the flail arm and flail leg ALS variants. Neurology 2009;72:1087-94.

10. Tanghe H. Magnetic resonance imaging (MRI) in syringomyelia. Acta Neurochirurg 1995;134:93-9.

11. Catala M. Réflexions d'un neuroembryologiste à propos de la syringomyélie dite malformative. Neuro-chirurgie Suppl1999;45:9-22.

12. Nishizawa S, Yokoyama T, Yokota N, et al. Incidentally identified syringomyelia associated with Chiari I malformations: is early interventional surgery necessary? Neurosurgery 2001;49:637-41.

13. Hu M, Ellis C, Al-Chalabi A, et al. Flail arm syndrome: a distinctive variant of amyotrophic lateral sclerosis. J Neurol Neurosurg Psych1998;65(6):950-51.

14. Gamez J, Cervera C, Codina A. Flail arm syndrome or VulpianBernhart's form of amyotrophic lateral sclerosis. J Neurol Neurosurg Psych 1999;67:2589.

15. Mulder DW. The clinical syndrome of amyotrophic lateral sclerosis.
Proceedings of the staff meetings Mayo Clinic; 1957.

16. Rowland LP, Shneider NA. Amyotrophic lateral sclerosis. New Engl J Med 2001;344:1688-700.

17. Alden TD, Ojemann JG, Park T. Surgical treatment of Chiari I malformation: indications and approaches. Neurosurg Focus 2001;11:1-5.

18. Todor DR, Mu HT, Milhorat TH. Pain and syringomyelia: a review. Neurosurg Focus 2000;8:1-6.

19. Paul KS, Lye RH, Strang FA, et al. Arnold-Chiari malformation: review of 71 cases. J Neurosurg 1983;58:183-7.

20. Ellenbogen RG. How Chiari malformation and syringomyelia are diagnosed. In: U. Batzdorf, E.C. Benzel, R.G. Ellenbogen, F.M. Ferrante, B.A. Green, A.H. Menezes, M.C. Speer, eds. Chiari malformation and syringomyelia. A handbook for patients and their families. Rocklin, CA: American Chronic Pain Association; 2008. pp. 13-16.

21. Milhorat TH, Chou MW, Trinidad EM, et al. Chiari I malformation redefined: clinical and radiographic findings for 364 symptomatic patients. Neurosurgery 1999;44:1005-17.

22. Dyste GN, Menezes AH, VanGilder JC.
Symptomatic Chiari malformations: an analysis of presentation, management, and long-term outcome. J Neurosurg 1989;71:159-68

23. Zamel K, Galloway G, Kosnik EJ, et al. Intraoperative neurophysiologic monitoring in 80 patients with Chiari I malformation: role of duraplasty. J Clin Neurophysiol 2009;26:70-5.

24. Novegno F, Caldarelli M, Massa A, et al. The natural history of the Chiari Type I anomaly. 2008

25. al. Transverse microincisions of the outer layer of the dura mater combined with foramen magnum decompression as treatment for syringomyelia with Chiari I malformation. Acta Neurohirurg 1998;140:134-9.

26. Holly LT, Batzdorf U. Management of cerebellar ptosis following craniovertebral decompression for Chiari I malformation. J Neurosurg 2001;94:21-6.

27. Isu T, Sasaki H, Takamura $H$, et al. Foramen magnum decompression with removal of the outer layer of the dura as treatment for syringomyelia occurring with Chiari I malformation. Neurosurgery 1993;33:845-50. 